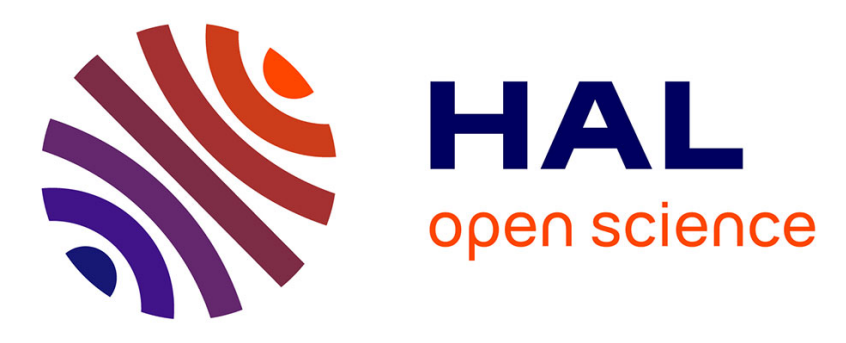

\title{
Poroelastic lamellar metamaterial for sound attenuation in a rectangular duct
}

Ke Li, Benoit Nennig, Emmanuel Perrey-Debain, Nicolas Dauchez

\section{To cite this version:}

Ke Li, Benoit Nennig, Emmanuel Perrey-Debain, Nicolas Dauchez. Poroelastic lamellar metamaterial for sound attenuation in a rectangular duct. Applied Acoustics, 2021, 176, pp.107862. 10.1016/j.apacoust.2020.107862 . hal-03112506

\section{HAL Id: hal-03112506 \\ https://hal.science/hal-03112506}

Submitted on 23 Feb 2021

HAL is a multi-disciplinary open access archive for the deposit and dissemination of scientific research documents, whether they are published or not. The documents may come from teaching and research institutions in France or abroad, or from public or private research centers.
L'archive ouverte pluridisciplinaire HAL, est destinée au dépôt et à la diffusion de documents scientifiques de niveau recherche, publiés ou non, émanant des établissements d'enseignement et de recherche français ou étrangers, des laboratoires publics ou privés. 


\title{
Poroelastic lamellar metamaterial for sound attenuation in a rectangular duct
}

\author{
Ke Li ${ }^{\mathrm{a}}$, Benoit Nennig ${ }^{\mathrm{b}}$, Emmanuel Perrey-Debain ${ }^{\mathrm{a}}$, Nicolas Dauchez ${ }^{\mathrm{a}, *}$ \\ ${ }^{a}$ Université de technologie de Compiègne, CNRS, Roberval, Centre de recherche Royallieu - CS 60319 - 60203 Compiègne Cedex, France \\ ${ }^{b}$ Institut supérieur de mécanique de Paris (SUPMECA), Laboratoire Quartz EA 7393, \\ 3 rue Fernand Hainaut, 93407 Saint-Ouen, France.
}

\begin{abstract}
The sound attenuation of a silencer consisting of a lamella network made with melamine foam inserted in rectangular duct is investigated numerically and experimentally. The lamella arrangement is designed so that skeleton bending elastic resonances appear at low frequency. In addition to this phenomenon, viscothermal losses in the porous material ensure broadband attenuation. The lamella network provides a natural sub-wavelength resonator, without any other kind of inclusions, and create an easy to manufacture metamaterial with high tunability and broadband efficiency. Experimental transmission losses compare well with three dimensional finite element model obtained either on the whole silencer, either on a periodic cell. A parametric study is conducted on the periodic model to identify the effect of different geometrical parameters, like dimensions of the lamella and air gap, as well as physical parameters, like air flow resistivity, elastic modulus and loss factor of the poroelastic material, on the sound attenuation in the silencer.
\end{abstract}

Keywords: Silencer, Poroelastic material, Metamaterial, Duct acoustics, Exceptional point, HVAC, Manufacturing, Periodic cell, Finite element model

\section{Introduction}

The control of low frequency noise remains a challenge for the automotive, aircraft and building industries. In many instance, the noise is produced by various airflow systems and is transmitted in ducts which act as acoustic waveguides.

The mitigation of these noise disturbances is usually accomplished using passive treatments by either using acoustic liners which consists in treating the wall of the duct adequately or by inserting dissipative splitter silencers in the duct. In the latter case, silencers are made with fibrous materials such as rock wool or glass fibre and are thus less effective at low frequency and for best sound attenuation, it is normally required that the thickness of the treatment should be of the same order as the acoustic wavelength [1-3]. Traditional acoustic liners, made of a resistive perforated plate coupled to a quarter-wavelength resonator, somewhat suffers from the same limitation as low frequency performances are typically limited by the cavity depth. The geometry structure of these liners, which consist of a periodic arrangement of acoustic resonators make these the direct ancestor of acoustic metasurfaces or metamaterials which have experienced a continuous and robust development for the last twenty years. Because these materials have effective dynamic quantities with negative values, like negative mass density [4] and bulk modulus $[5,6]$, that can not be observed in natural

\footnotetext{
${ }^{*}$ Corresponding author

Email addresses: ke.li@utc.fr (Ke Li),

benoit.nennig@supmeca.fr (Benoit Nennig),

emmanuel . perrey-debain@utc.fr (Emmanuel Perrey-Debain),

nicolas.dauchez@utc.fr (Nicolas Dauchez)
}

materials, they break the traditional design rules for acoustic treatment.

The corner stone of these materials relies on subwavelength resonators and its associated phase shift. Here, we can mention quarter wavelength and space-coiling structures [7-9], Helmholtz resonators [6, 10-12] or membranes [4, 13]. The geometrical configuration of the resonators network can also play a crucial role, like in slow sound channels $[14,15]$. Another major ingredient which must be considered in order to design efficient sound absorbing materials is the losses. Viscothermal losses in porous materials [16] have been combined with the inclusion of small resonators in the so-called metaporous materials [12, 17-20], showing improvement at low frequency while keeping broadband absorption. Perfect absorption can also be achieved by the mechanism of critical coupling whereby the leakage rate of energy out of the resonator and its inherent losses are properly balanced [13].

Recently, Christensen et al. [21] proposed a structured material fabricated out of porous lamellas backed by a reflecting support. The increase in dissipation is explained by the fact that sound is trapped more efficiently than for a homogeneous porous layer. It is reported that complete absorption of sound within a two octave band can be obtained though the concept is not optimal within the long wavelength regime and at grazing incidence. Dauchez et al. [22] studied sound absorption of a large scale poroelastic lamella network under oblique incidence in free field. Results show that this type of structured material, which bears similarity with [21], permits to gain extra absorption in the low frequency range by taking advantage of the resonance of the elastic frame of the foam. This particular effect which is often ignored in the literature is indeed not 
studied in [21].

Silencers and sound attenuation in waveguides by metamaterial have been less investigated than for panels dedicated to acoustic room corrections. The grazing incidence of sound waves which, depending on the duct dimensions, may propagate in a multimode context makes the design of appropriate acoustic treatments more complex and less understood.

Generally the best attenuation is obtained when two guided modes of the silencer are close to merge [23-26]. This can be achieve by exploiting Fano resonances [27, 28] or the high tunability of metamaterial [24, 25]. Another lever is to take advantage of poroelastic frame elastic resonances. Their strong impact on the sound attenuation have been shown in [29-31] in the poroelastic silencer.

The present paper is in the wake of previous work published by the authors $[22,29,30]$ on the role of the frame elasticity for the passive sound control. More precisely, our aim is to devise and investigate a new duct silencer concept made of a specific arrangement of lamellas, here made of melamine foam, inserted in a rectangular duct. The lamella bending motion provides a natural sub-wavelength resonator, without any other inclusions. The interest of this configuration rely on its simple manufacturing, its high tunability and its broadband efficiency.

The paper is organized as follows. First, the experimental setup and material properties are described. Two configurations with different orientations of the lamellas, i.e. parallel or perpendicular to the duct axis, are investigated and compared to the homogeneous case.

Experimental results are given in terms of the Transmission Losses (TL) of the silencer and are compared with numerical simulations obtained either from a full model, i.e. a 3D finite element model (FEM) of the whole silencer, and from a simplified and idealized periodic FEM model. This simplified model is then used to carry out a parametric study in order to identify the effect of different geometrical parameters as well as physical parameters such as the airflow resistivity and the Young elastic modulus of the poroelastic material. Taking advantage of the modal description given by periodic model, the paper ends with a discussion which highlights the fact that best attenuation are nearly-optimal when modes are close to veering condition. This phenomena which was established earlier by Tester [23] for locally reacting liners is also described in recent papers [25, 26].

\section{Experimental approach}

\subsection{Description of the poroelastic lamellar metamaterial}

The poroelastic lamellar metamaterial shown in Fig. 1 is made up of several melamine foam strips which properties are given in Table 1. Each lamella has a nominal thickness $h_{1}=25$ $\mathrm{mm}$ along the $y$-axis, a width $w_{1}=15 \mathrm{~mm}$ and a length of 200 $\mathrm{mm}$. The air gap between two lamellas is $5 \mathrm{~mm}$. There are ten parallel strips glued on a $20 \mathrm{~cm} \times 20 \mathrm{~cm}$ stiff plate. Four samples are fabricated and arranged with different orientations. The two configurations investigated, parallel and perpendicular, are shown in Fig. 2. In the parallel arrangement, with 10 lamellas, each lamella is parallel to the duct axis, which is also

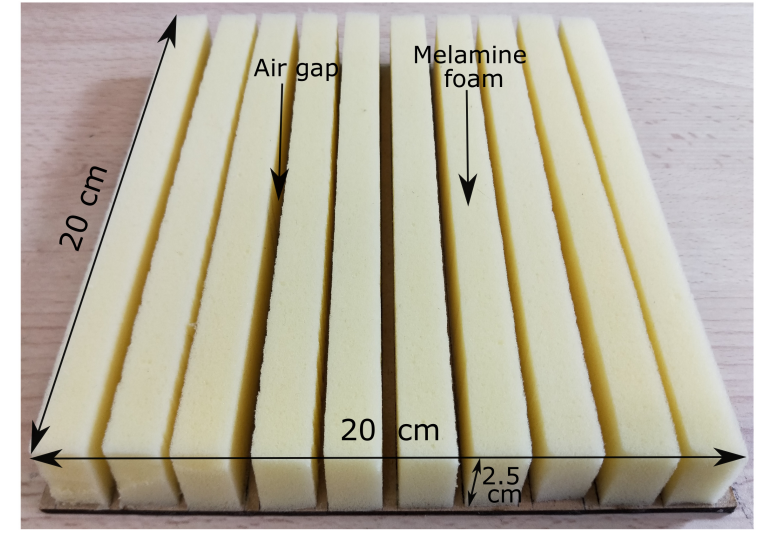

Figure 1: Poroelastic lamellar metamaterial.

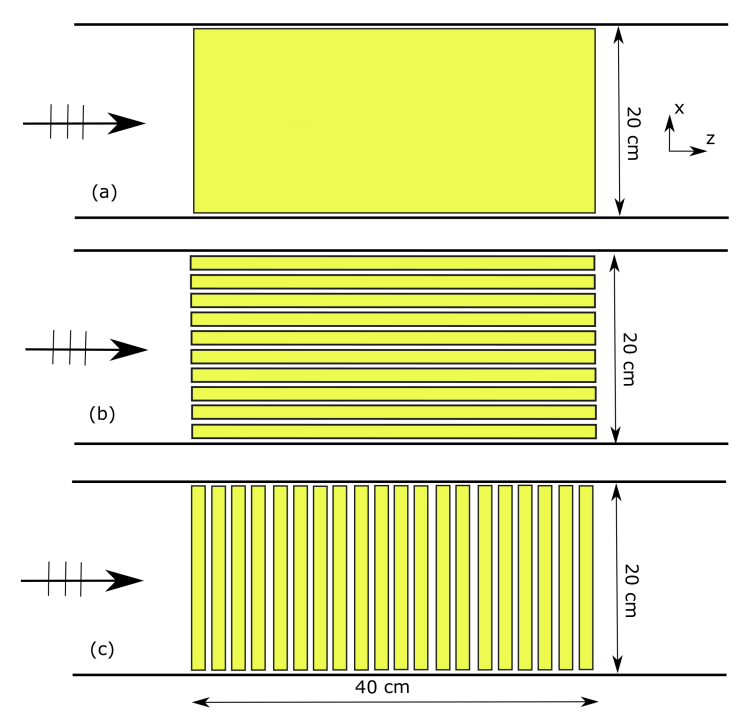

Figure 2: Orientations of the lamellar material; (a): homogeneous; (b): parallel; (c): perpendicular.

the direction of the incident pressure field. In the perpendicular arrangement, with 20 lamellas, each lamella is perpendicular to the duct axis. In all cases, the sample covers both bottom and top surfaces of the duct over a $40 \mathrm{~cm}$ length in the $z$-direction (see Fig. 3).

\subsection{Experimental setup}

The experimental set-up used to measure the Transmission Loss (TL) of the lined duct is also shown. The test bench has been designed for the acoustic multi-modal characterization of a test section in presence of a low Mach number flow within the frequency band [200 Hz-3.5 kHz]. In this work, we consider the no flow case and only the incident plane wave is accounted for. The duct has a rectangular section of $0.2 \mathrm{~m} \times 0.1$ $\mathrm{m}$ with an anechoic termination at both ends. The scattering matrix, which contains the modal reflection and transmission 


\begin{tabular}{|c|c|c|c|c|c|c|c|c|}
\hline $\begin{array}{c}\text { Porosity } \\
\phi\end{array}$ & $\begin{array}{c}\text { Airflow resistivity } \\
\sigma \\
\left(\mathrm{Nm}^{-4} \mathrm{~s}\right)\end{array}$ & $\begin{array}{c}\text { Viscous length } \\
\Lambda \\
(\mu \mathrm{m})\end{array}$ & $\begin{array}{c}\text { Thermal length } \\
\Lambda^{\prime} \\
(\mu \mathrm{m})\end{array}$ & $\begin{array}{c}\text { Tortuosity } \\
\alpha_{\infty}\end{array}$ & $\begin{array}{c}\text { Density } \\
\rho_{1} \\
\mathrm{~kg} \cdot \mathrm{m}^{-3}\end{array}$ & $\begin{array}{c}\text { Young's modulus } \\
E \\
\mathrm{kPa}\end{array}$ & $\begin{array}{c}\text { Loss factor } \\
\eta\end{array}$ & $\begin{array}{c}\text { Poisson ratio } \\
v\end{array}$ \\
\hline 0.982 & 7920 & 132.6 & 149.9 & 1 & 6.11 & 120 & 0.075 & 0 \\
\hline
\end{tabular}

Table 1: Properties of the poroelastic material [22].
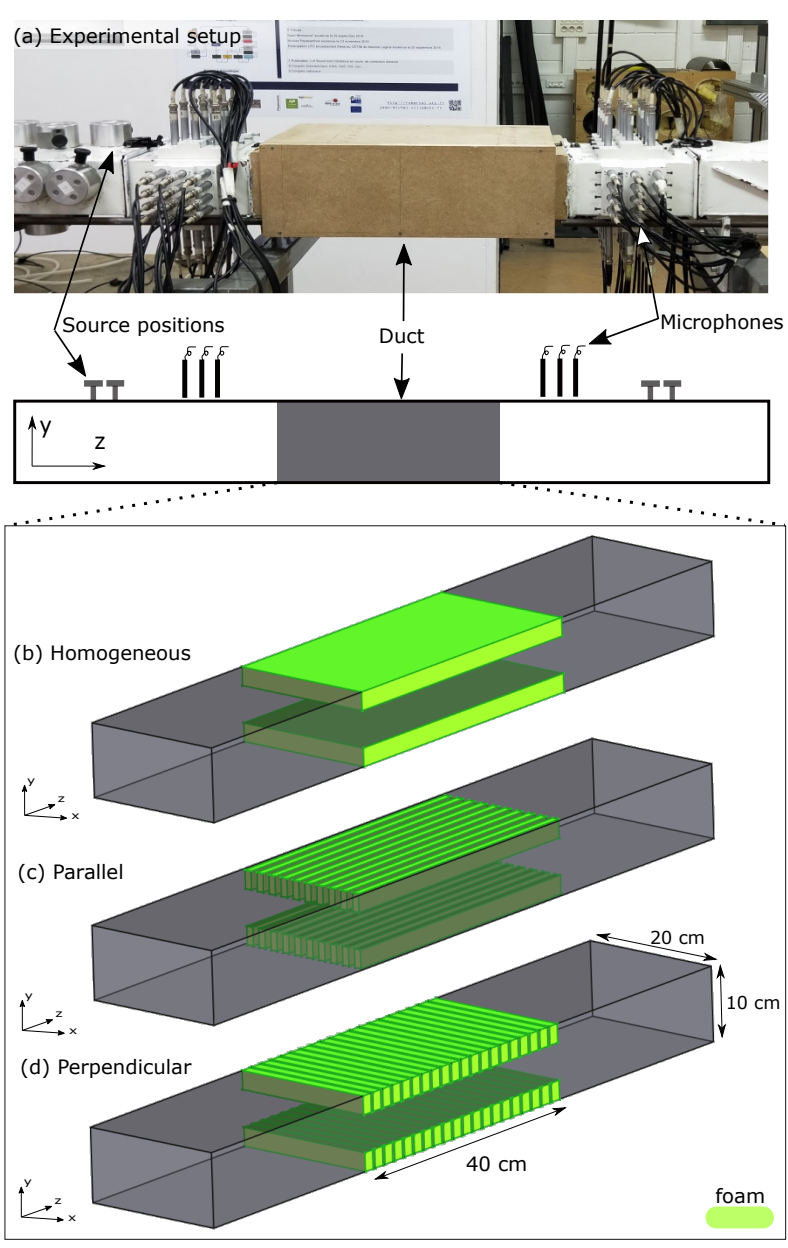

Figure 3: Experimental setup for transmission loss measurement (a) and lamellas orientations (b-d).

coefficients is measured using a multi-source method described in [32]. Note that the symmetry of each configuration tested prevents the existence of the first transverse duct acoustic mode so in the frequency range of interest, here up to $1500 \mathrm{~Hz}$, only the plane wave mode is allowed to propagate in the rigid duct.

\subsection{Results}

In order to illustrate the influence of the orientation and of the filling fraction, parallel and perpendicular orientations are compared to an homogeneous layer made of the same material. These results are given in Fig. 4. The TL obtained with the homogeneous layer exhibits a peak around $850 \mathrm{~Hz}$. Attenuation peaks can be related to the cutoff frequency of Lamb-

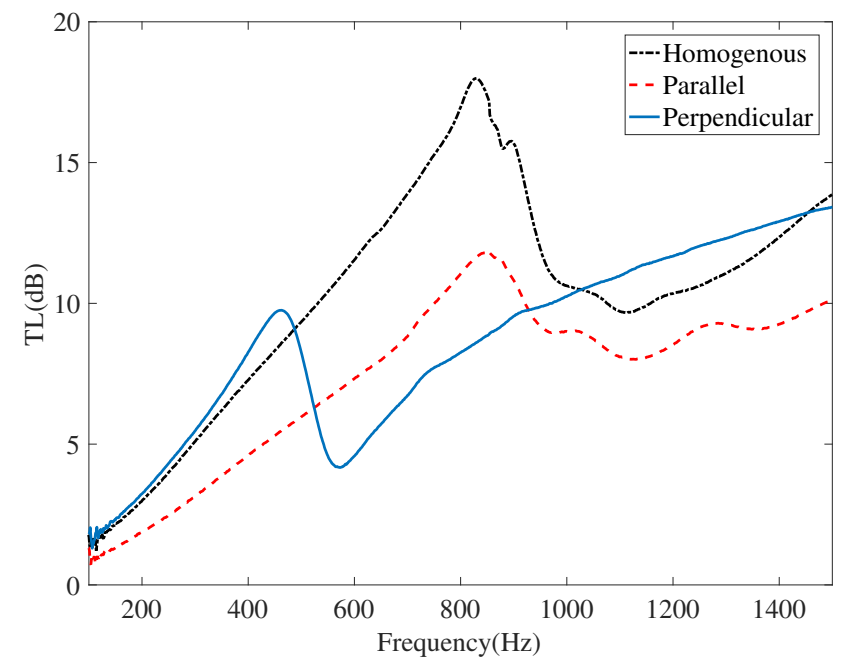

Figure 4: Comparison of experimental results for parallel (- -), perpendicular(_) and homogeneous layer (...).

like waves. Because of the motionless boundary condition on the bottom, all elastic modes in the slab problem have a cutoff frequency, whereas classical Lamb waves are associated with pressure release conditions on both sides. Exact solutions for fully coupled systems can be found numerically in [29, 33]. It is sufficient in the present analysis to remind the following approximation which holds if the coupling with the fluid is neglected [33]:

$$
f_{i, m} \approx(2 m+1) \frac{c_{i}}{4 h_{1}}, \text { for } i=s, l
$$

where $c_{s}=\sqrt{\frac{E / 2(1+v)}{\rho_{1}}}$ and $c_{l}=\sqrt{\frac{E(1-v) /[(v+1)(1-2 v)]}{\rho_{1}}}$ are the in vacuo shear and longitudinal bulk velocity, respectively. These cutoff frequencies correspond to quarter wavelength (or its multiple) resonance of the shear and compression wave of an homogeneous layer, as mentioned in [22] for absorbing panels.

The parallel configuration exhibits the same trend, with a lower TL due to the air gaps that increase the macro-porosity of the sample.

The perpendicular configuration exhibits also a peak, but at a lower frequency around $440 \mathrm{~Hz}$, resulting from the excitation of the first bending resonance of the lamella [22]. Based on a simple cantilever beam model, its first frequency can be approximated by

$$
f_{b} \approx 0.56 \frac{w_{1}}{h_{1}^{2}} \sqrt{\frac{E}{12 \rho_{1}}} .
$$




\section{Numerical models}

\subsection{Model of the silencer}

In order to further interpret experimental results and analyze absorption mechanisms taking place in the porous material, the wave propagation in the silencer is computed numerically using Finite Element Method. Typical FEM meshes used in our calculations are illustrated in Fig. 5 showing the poroelastic domain $\Omega_{p}$ and the air domain $\Omega_{a}$ for the three configurations. The dimensions of the duct and the silencer are the same as the experimental one.

In the air domain of density $\rho_{0}$ and sound speed $c_{0}$, the acoustic pressure $p$ obeys the Helmholtz equation

$$
\Delta p+k_{0}^{2} p=0
$$

where $k_{0}=\omega / c_{0}$ is the wavenumber (time dependence $e^{-\mathrm{i} \omega t}$ is considered here). On the rigid wall, the acoustic normal velocity vanishes. In the porous domain $\Omega_{p}$, the classical mixed $\left(\mathbf{u}, p_{p}\right)$ formulation [16, Chap. 13] is used as it allows to reduce the number of degrees of freedom and permits to ease the treatment of the transmission conditions at the air-porous interface. The formulation is reminded here:

$$
\begin{aligned}
\nabla \cdot \hat{\boldsymbol{\sigma}}^{s}(\mathbf{u})+\omega^{2} \rho \mathbf{u}+\gamma \nabla p_{p} & =0, \\
\Delta p_{p}+\omega^{2} \frac{\rho_{22}}{R} p_{p}-\omega^{2} \frac{\rho_{22}}{\phi^{2}} \gamma \nabla \cdot \mathbf{u} & =0 .
\end{aligned}
$$

Here, $p_{p}$ is the pore pressure, $\phi$ is porosity of the porous material, $\gamma=\phi\left(\frac{\rho_{12}}{\rho_{22}}-\frac{Q}{R}\right)$ and $\rho=\rho_{11}-\frac{\rho_{12}^{2}}{\rho_{22}}$. Coefficient $R$ is the effective bulk modulus of the fluid phase and takes into account the thermal dissipation, $Q$ couples the two phases by volumic dilatation. The effective density coefficient $\rho_{11}$ and $\rho_{22}$, respectively for the solid phase and the fluid phase, and the coupling density coefficient $\rho_{12}$ are complex-valued, and their imaginary part takes into account viscous losses. The first two terms in (4a) and in (4b) describes respectively the dynamics of the elastic skeleton and equivalent fluid. The last term in both equations couples the two phases. The in vacuo stress tensor $\hat{\sigma}^{s}$ is given by

$$
\hat{\boldsymbol{\sigma}}^{s}(\mathbf{u})=\mathbf{I}\left(K_{b}-\frac{2}{3} N\right) \nabla \cdot \mathbf{u}+2 N \boldsymbol{\varepsilon}^{s}(\mathbf{u}) .
$$

Here, $K_{b}$ is the complex dynamic bulk modulus of the frame, $N$ is the shear modulus and includes the structural damping. All these coefficients are related to the poroelastic structural parameters (see Table 1) by the Johnson-Champoux-Allard model and can be found in Ref. [16, Chap. 6]. At the interface between the fluid and the porous material, the coupling conditions impose the continuity of normal displacement, of the pressure and normal stress (see for instance [16, Chap. 13]). Since the poroelastic material is clamped, the skeleton and the normal fluid displacements vanish on the duct wall. Radiation conditions at both ends of the duct and the incident pressure field have been implemented using the Dirichlet-to-Neumann (DtN) map [34] using the expansion of the pressure in terms of duct acoustic modes (see Appendix A). Duct modes are also used

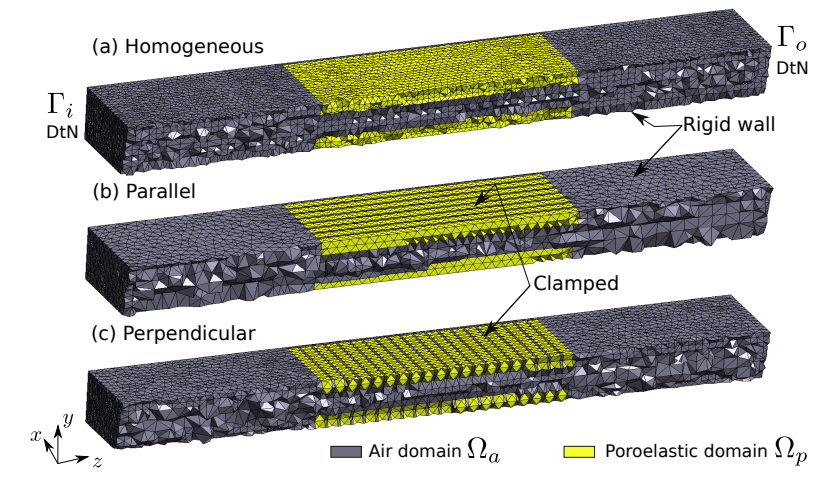

Figure 5: Meshes and boundary conditions for the three tested configurations: homogeneous (a), parallel (b), perpendicular (c).

to compute the The transmission loss (TL), defined as the ratio between the transmitted and incident power.

For completeness, the final form for the weak formulation is reminded in the Appendix B. Note that the pressure, in the air and in the pore, and solid displacements are discretized using Lagrange quadratic finite elements. In all cases, the mesh size was chosen to ensure a good trade-off between accuracy and computational time.

\subsection{Periodic model}

Here we shall use the periodic structure of the silencer and consider a single periodic cell. The two configurations, parallel and perpendicular, are depicted in Fig. 6. A typical cell of dimensions $w, d$ and $h$ is periodic in the $x$ and $z$ directions whereas rigid wall conditions are imposed at $y=0, h$ and, by invoking symmetry reasons, we take $h=5 \mathrm{~cm}$ which corresponds to the half-width of the rectangular duct. The dimension $d$ is arbitrary here and we take $d=6 \mathrm{~cm}$. Dimensions of the porous material are $w_{1} \times h_{1} \times d$ (recall that experimental values are $w_{1}=1.5$ $\mathrm{cm}$ and $h_{1}=2.5 \mathrm{~cm}$ ). The periodic model not only allows to diminish drastically the computational burden but also provides a new insight into the absorbing properties of the silencer with a view to optimisation, this will be exploited in Section 4.

The starting point stems from the Bloch theorem that states that all fluctuations, i.e. pressure and solid displacement, call it $X$, can be written as [35]

$$
X(\mathbf{x})=\hat{X}(\mathbf{x}) e^{i \mathbf{k}_{B} \cdot \mathbf{x}}, \text { for } \mathbf{x}=[x, y, z]^{t},
$$

where $\hat{X}$ is a periodic function with $\hat{X}(\mathbf{x}+\mathbf{d})=\hat{X}(\mathbf{x})$ where $\mathbf{d}=$ $[w, 0, d]^{t}$ and $\mathbf{k}_{B}$ is the Bloch wavevector which can also be expressed as $\mathbf{k}_{B}=k_{B} \boldsymbol{\kappa}$ with unit vector $\boldsymbol{\kappa}=\left[\kappa_{x}, \kappa_{y}, \kappa_{z}\right]^{t}$ and the norm $k_{B}=\left\|\mathbf{k}_{B}\right\|$. Thus, Bloch waves are $\mathbf{d}$-periodic functions modulated by plane waves involving the Bloch wavevector. The real part of $\mathbf{k}_{B}$ is the phase change across the cell, and more important, the imaginary part is related to the wave attenuation. Each configuration, i.e. parallel and perpendicular, is easily obtained by simply setting $\boldsymbol{\kappa}=[0,0,1]^{t}$ or $\boldsymbol{\kappa}=[1,0,0]^{t}$, respectively. 
(a) Parallel

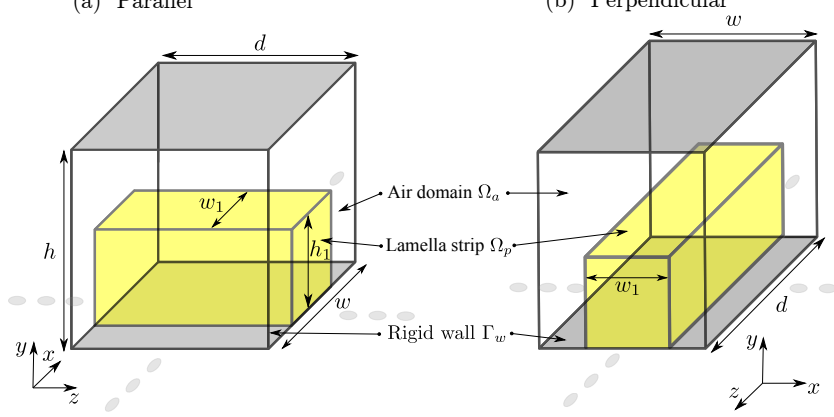

Figure 6: Periodic cell model; (a): perpendicular case; (b): parallel case

To solve the periodic problem with the FEM, the sytem of equations need to be rewritten for the periodic field $\hat{X}$. This can be done in a systematic way, from the original equations Eq. (3) and Eq. (4). For instance, in the air domain $\Omega_{a}$, using the expansion (6) yields the new wave equation

$$
\Delta \hat{p}+2 \mathrm{i} k_{B} \boldsymbol{\kappa} \cdot \nabla \hat{p}+\left(k_{B}^{2}-k_{0}^{2}\right) \hat{p}=0 .
$$

The wave equation in the poroelastic domain can be derived and a similar manner. The associated weak formulation is presented in Appendix B. Finally, the problem takes the form of a quadratic eigenvalue problem with eigenvalue $k_{B}$,

$$
\left[\mathbf{K}_{0}(\omega)+k_{B} \mathbf{K}_{1}(\omega)+k_{B}^{2} \mathbf{K}_{2}(\omega)\right] \hat{\mathbf{X}}=0,
$$

where $\hat{\mathbf{X}}$ contains the FEM unknowns and $\mathbf{K}_{i}(i=0,1,2)$ are FEM matrices detailed in Appendix B. The computation of Eq. (8) is performed after transformation into a generalized eigenvalue problem and the latter is solved using standard sparse solver libraries. The TL of the silencer is estimated, by assuming that (i) the lowest attenuated Bloch wave should provide a fair description of the wave field (both in the air and poroelastic domains) as it propagates in the silencer and (ii) the reflected waves at the entrance of the silencer can be neglected, and thus

$$
\mathrm{TL} \approx 8.68 \operatorname{Im}\left(k_{B}^{0}\right) L,
$$

where $k_{B}^{0}$ corresponds to the eigenvalue of Eq. (8) with smallest imaginary part and $L=0.4 \mathrm{~m}$ is the length of the silencer.

\subsection{Comparison with experimental results}

The TL of the three configurations are compared in Fig. 7 showing satisfactory agreement between numerical, here using the full FEM model, and experimental results. A convenient way to determine more precisely the influence of the solid frame is to investigate the relative contribution of the different dissipation mechanisms involved in the sound attenuation. Three mechanisms are considered: viscous, thermal and structural $[36,37]$. Viscous loss is due to the viscosity of the air and the relative movement air-skeleton in the porous material. Thermal dissipation is due to the heat exchange between the air
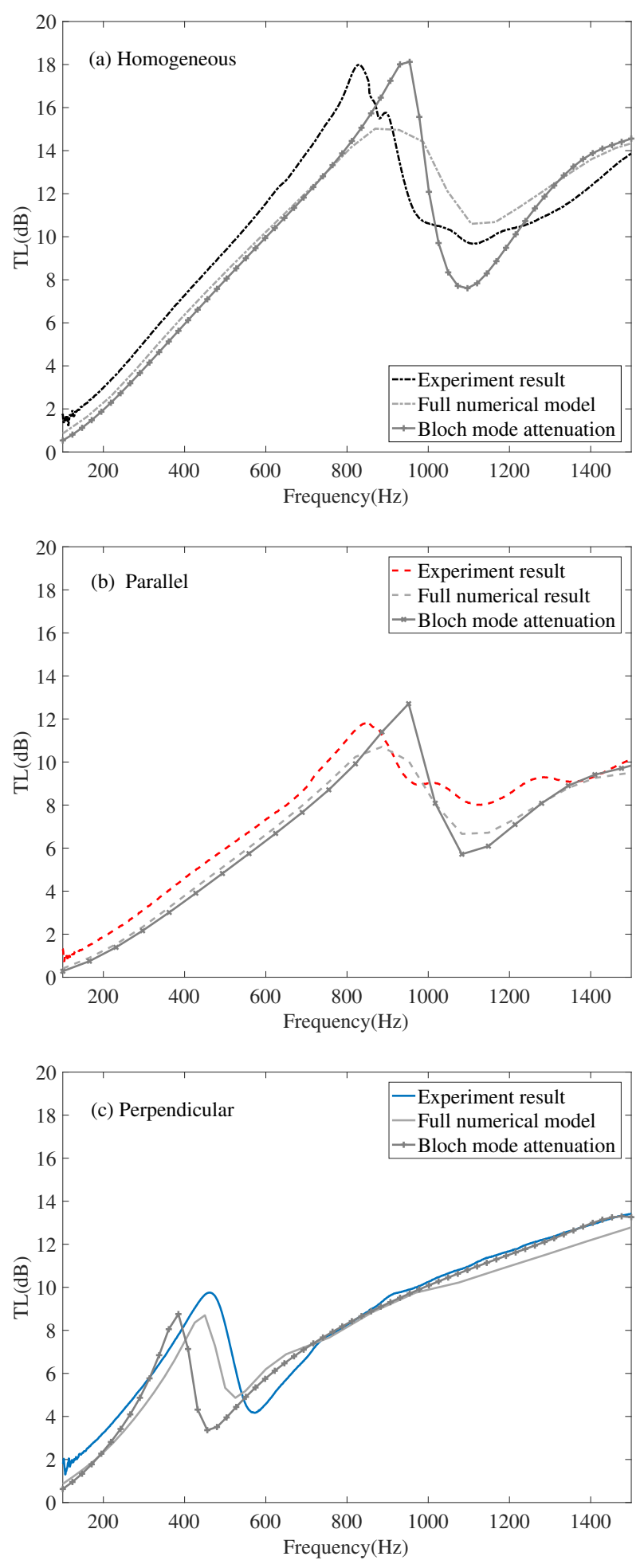

Figure 7: Comparison of experimental (black dashed line) and numerical (black solid line) results for the three configurations: (a): homogeneous, (b): parallel and (c): perpendicular 
(a) $u_{z}$ on parallel case

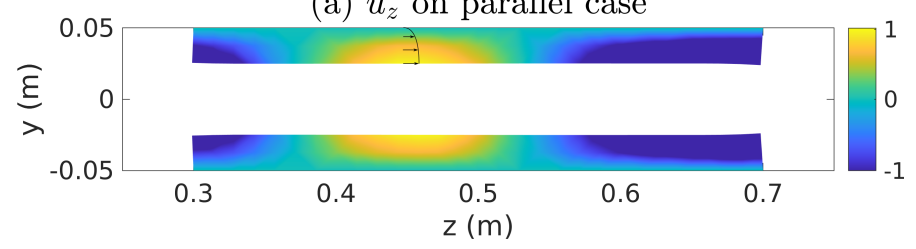

(b) $u_{z}$ on perpendicular case

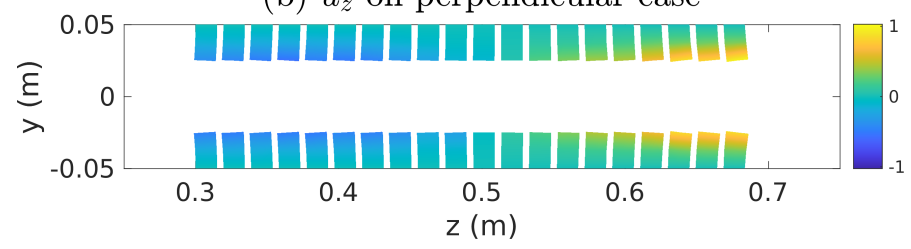

Figure 8: Displacement fields (color and motion) in the poroelastic material: (a) parallel configuration at shear resonance around $900 \mathrm{~Hz}$; (b) perpendicular configuration at bending resonance around $450 \mathrm{~Hz}$. Normalized color scale.

and the skeleton. Structural dissipation is due to the viscoelasticity of the skeleton and its strain energy. Results are presented for the three configurations in Fig. 9. Generally through the whole range of frequency, the viscous dissipation is dominating and has similar trend. Thermal dissipation is the second important attenuation mechanism. Structural dissipation is generally smaller except close to the frame resonances, around $900 \mathrm{~Hz}$ for the parallel and homogeneous configurations where the first shear resonance occurs. For the perpendicular configuration, the structural dissipation reaches its maximum around $450 \mathrm{~Hz}$ which corresponds to the first bending mode of the lamellas and is more pronounced. Thermal dissipation is found to increase steadily with frequency and is less affected by the frame resonance.

Clearly, the additional enhancement in sound attenuation due to the frame resonance, which can not exist with rigid frame porous materials, offers an interesting alternative for low frequency noise control. The resonance frequency of the first bending mode of the poroelastic lamella is simply determined by its dimensions, Young's modulus and density, thus allowing to design tailored solutions as shown in Ref. [22]. To have a better understanding of the physical mechanisms taking place, it is instructive to illustrate the horizontal displacement field of the skeleton, see Fig. 8, when the skeleton of the silencer resonates. For the parallel configuration, one sees the shear motion along one lamella, and for the perpendicular configuration, the bending motion of each lamella. The color allows to see the phase shift and the wavelength close to the acoustic wavelength that is $38 \mathrm{~cm}$ at $900 \mathrm{~Hz}$ and $75 \mathrm{~cm}$ at $450 \mathrm{~Hz}$.

Finally, using the periodic model, Bloch waves attenuations calculated from Eq. (9) are also shown in Fig. 7. Comparisons with the full model show very good agreements and the skeleton resonances are well captured. Small discrepancies stem mainly from the reflected waves at the entrance of the silencer which are not taken into account in the simplified model. In addition,
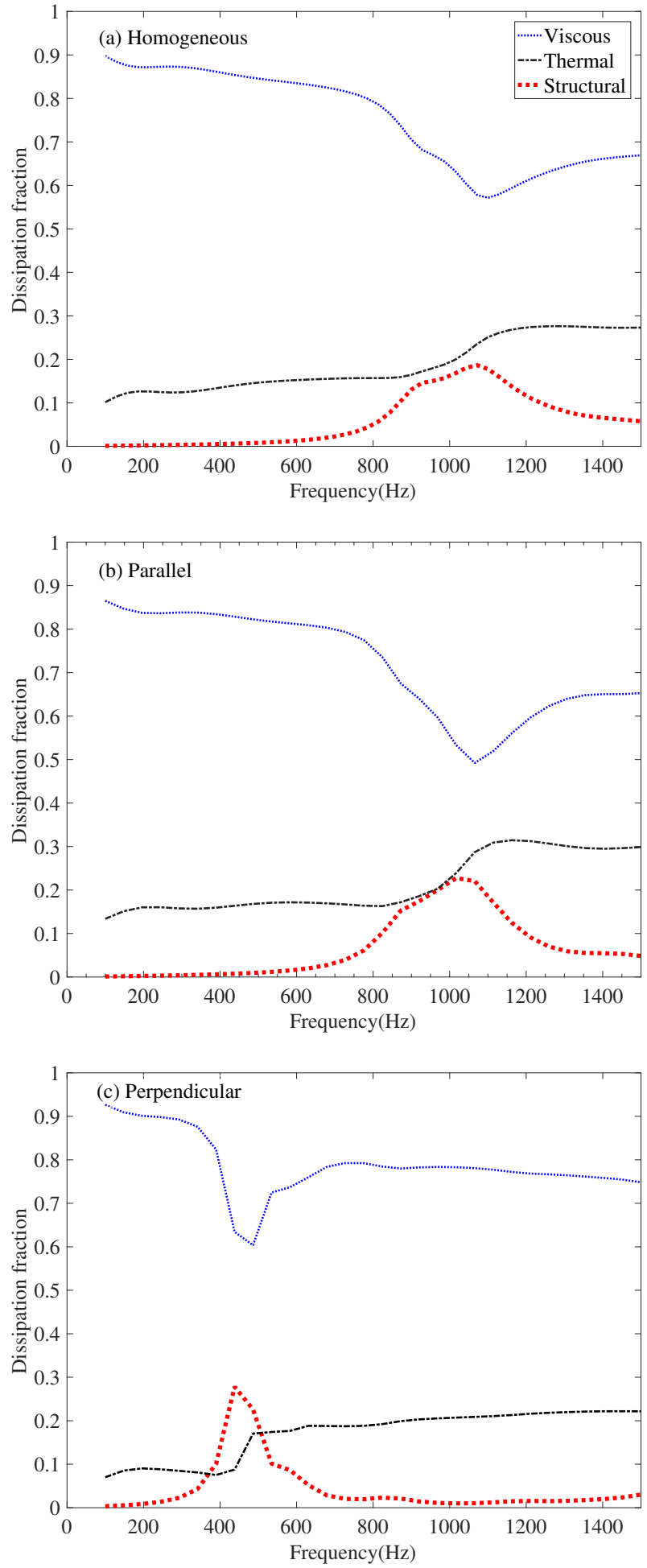

Figure 9: Contribution of viscous (blue dashed line), thermal (black dash-dotted line) and structural dissipation (red dashed line) for the three configurations: (a) homogeneous layer, (b) parallel and (c) perpendicular. 
the reduced periodic FEM model allows thinner mesh that better capture the skeleton motion.

\section{Parametric study}

In this section, we shall benefit from the last observations and analyze the efficiency of the silencer with the periodic model, thus avoiding the computational burden of the full model. Our aim is to conduct a parametric study and show the influence of some parameters, geometrical and physical which can be gathered in two groups. First, we study parameters related to the bending resonance, that is the Young's modulus, the lamella's width and the structural damping. Then, we study the influence of air-skeleton coupling parameters, characterized by the air gap width and the air flow resistivity.

\subsection{Bending resonance related parameters Young modulus}

Fig. 10 shows the effect of Young's modulus on the attenuation for both parallel (a) and perpendicular configurations (b). The nominal value is $E=120 \mathrm{kPa}$. Resonance peaks, which can be anticipated using the approximation Eq. (1) and (2) for parallel and perpendicular configurations respectively, is proportional to the square root of the Young's modulus $E$ as can be observed. The loss factor is constant in the analysis so the attenuation also increases with $E$ near the resonance.

\section{Width of lamella}

The width of lamella $w_{1}$ (see Fig. 6) is modified from $6 \mathrm{~mm}$ to its nominal value $15 \mathrm{~mm}$, keeping the air-gap $w-w_{1}$ constant. The results are shown in Fig. 11. For the parallel configuration (Fig. 11a), the location of the peak is not affected as expected from the theoretical estimate Eq. (1). However, the attenuation increases with $w_{1}$ since the filling fraction of the porous liner increases and tends to behave like the homogeneous configuration. For the perpendicular configuration (see Fig. 11b), the frequency of the peak increases almost linearly with $w_{1}$, according to Eq. (2). This means that it is possible to match a specific frequency by merely choosing the width of the lamella. Of course reducing the width has a negative impact on the attenuation but this can be partly limited by reducing the width of air gap as shown later.

\section{Loss factor}

The effect of the loss factor is shown in Fig. 12. Reducing the loss factor tends to produce a higher peak of attenuation followed by a more pronounced dip, and this is observed for both configurations. A too large loss factor will reduce the motion of the lamella at the resonance and therefore diminishes the associated additional dissipation.

\subsection{Air-skeleton coupling related parameters}

The effective coupling bewteen the air and the porous frame plays an important role in the sound attenuation of the lamella network. The effect of air gap width and air flow resistivity, which is the most influential coupling parameter in the low frequency range as it is related to viscous dissipation, are now investigated.
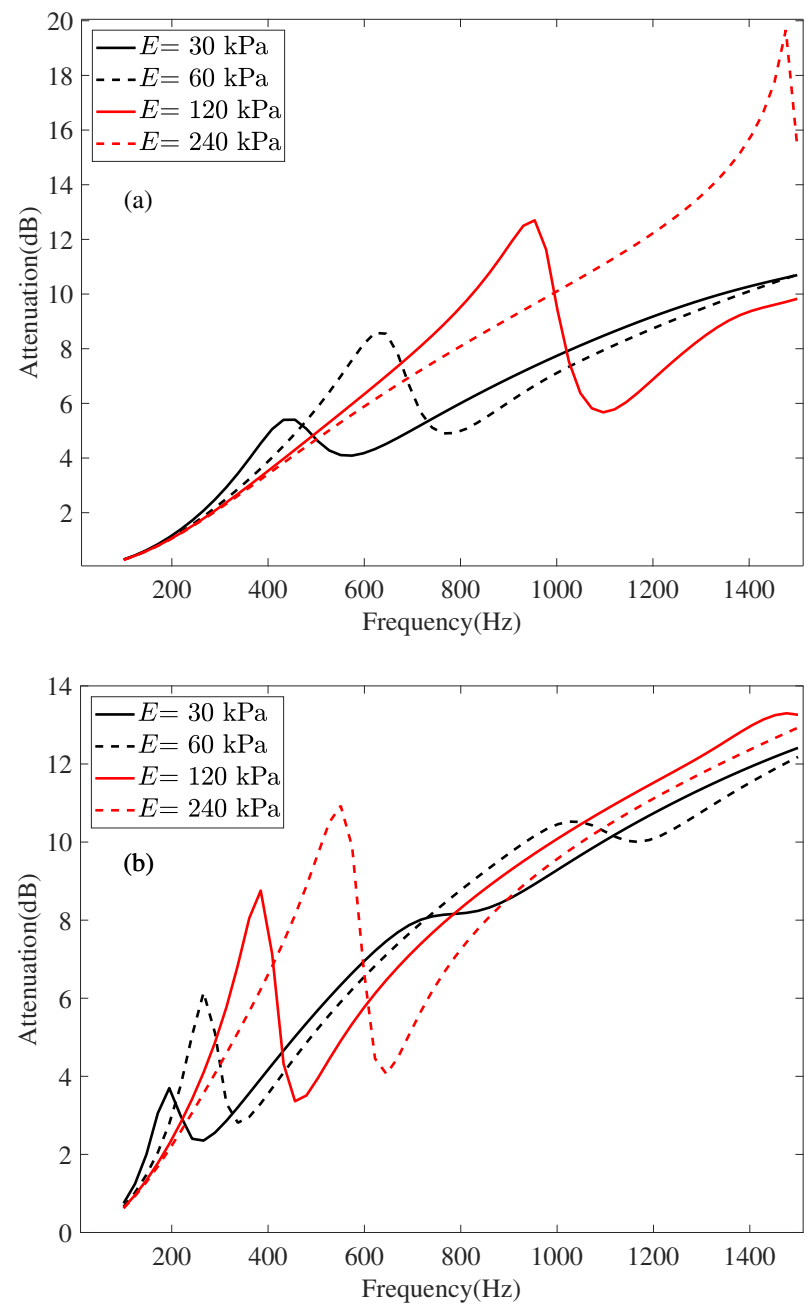

Figure 10: Comparison of TL performance for lamella network with different modulus from 30000 to $240000 \mathrm{~Pa}$. For parallel (a) and perpendicular (b) configurations.

\section{Air gap width}

We study the effect of the air gap $w-w_{1}$ ranging from 1 $\mathrm{mm}$ to $10 \mathrm{~mm}$, keeping the lamella width constant $w_{1}=15$ $\mathrm{mm}$. Reducing the air gap increases the volume of sound absorbing material. This can be observed in Fig. 13 in both configurations. Note that the air gap width has a stronger impact at the resonance for the parallel configuration, showing a variation of $8 \mathrm{~dB}$ whereas effects on the perpendicular configuration are more moderate. This can be explained by the different coupling mechanism between the movement of air and the porous frame also to the fact that the bending resonance occurs at a lower frequency.

\section{Airflow resistivity}

The airflow resistivity is known to be the most important parameter governing the acoustic dissipation in the low frequency range [16]. In the specific case of a silencer made up of a number of parallel splitters, there exists an optimal value for the resistivity as explained in [3] for splitters silencer. In the present 

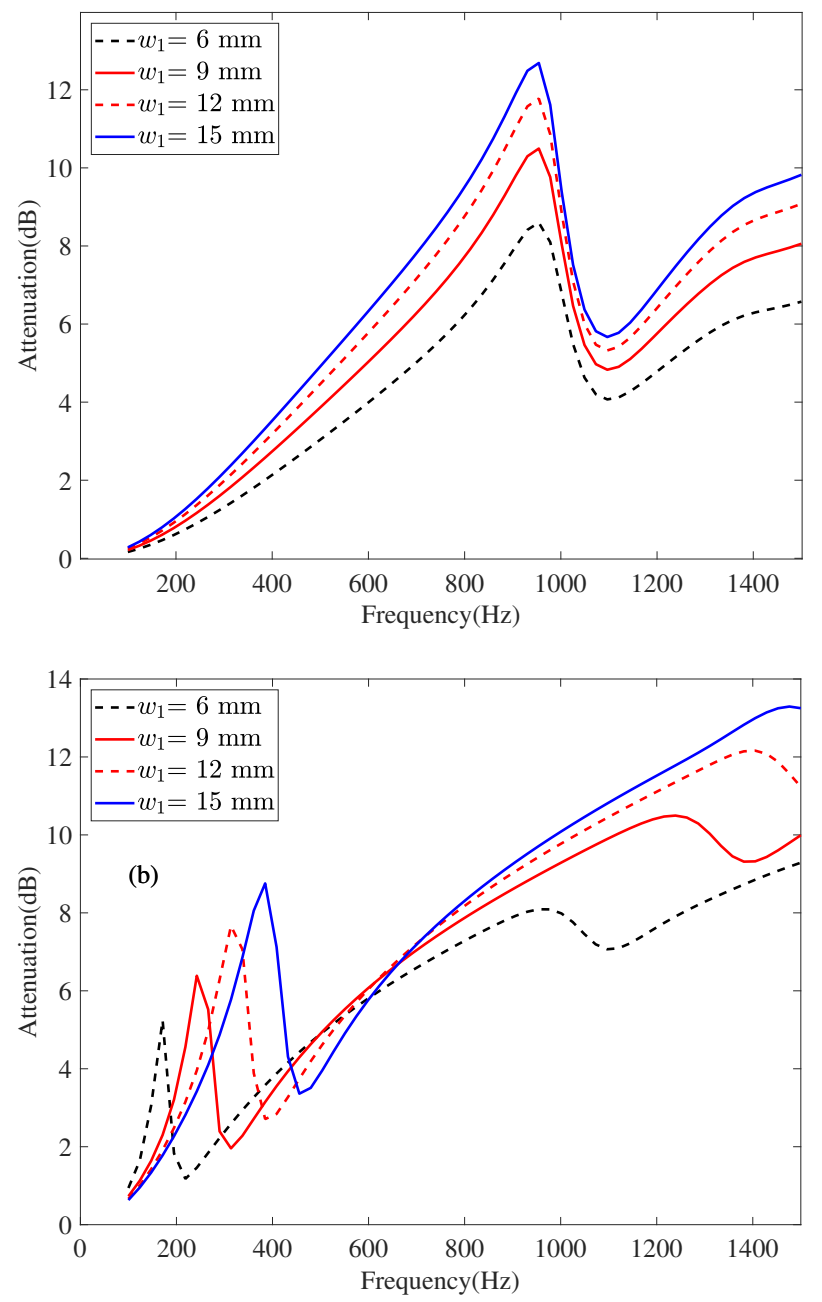

Figure 11: Attenuation for different lamella width $w_{1}=[6 \mathrm{~mm}$, $9 \mathrm{~mm}, 12 \mathrm{~mm}, 15 \mathrm{~mm}$ ]. parallel (a); perpendicular (b).

work, the fact is also observed in Fig. 14, showing the attenuation of silencer for different values ranging from $5000 \mathrm{Nm}^{-4} \mathrm{~s}$ to a very high value of $320000 \mathrm{Nm}^{-4} \mathrm{~s}$. In the parallel configuration, which somewhat bears resemblance with the configuration considered in [3], the attenuation at the resonance shows strong variations, up to $20 \mathrm{~dB}$. Here the maximum value, above 25 $\mathrm{dB}$, has been found to be nearly optimal. For the perpendicular configuration, the attenuation at the first bending resonance increases with $\sigma$ from $7 \mathrm{~dB}$ to $22 \mathrm{~dB}$. In this configuration, the optimal value at the peak can reach a higher value to the detriment of a poorer attenuation over a larger frequency spectrum.

\subsection{Towards an optimized configuration}

The parametric study made earlier permits a physical interpretation of the different mechanisms related to the acoustic attenuation. It is also instructive to address the problem from a mathematical point of view by observing that the quadratic eigenvalue problem of Eq. (8) is non-Hermitian due the dissipative nature of the media. In the context of guiding waves, it has been observed in many instances that optimal modal attenuation occurs when two eigenvalues, here the Bloch wavenum-
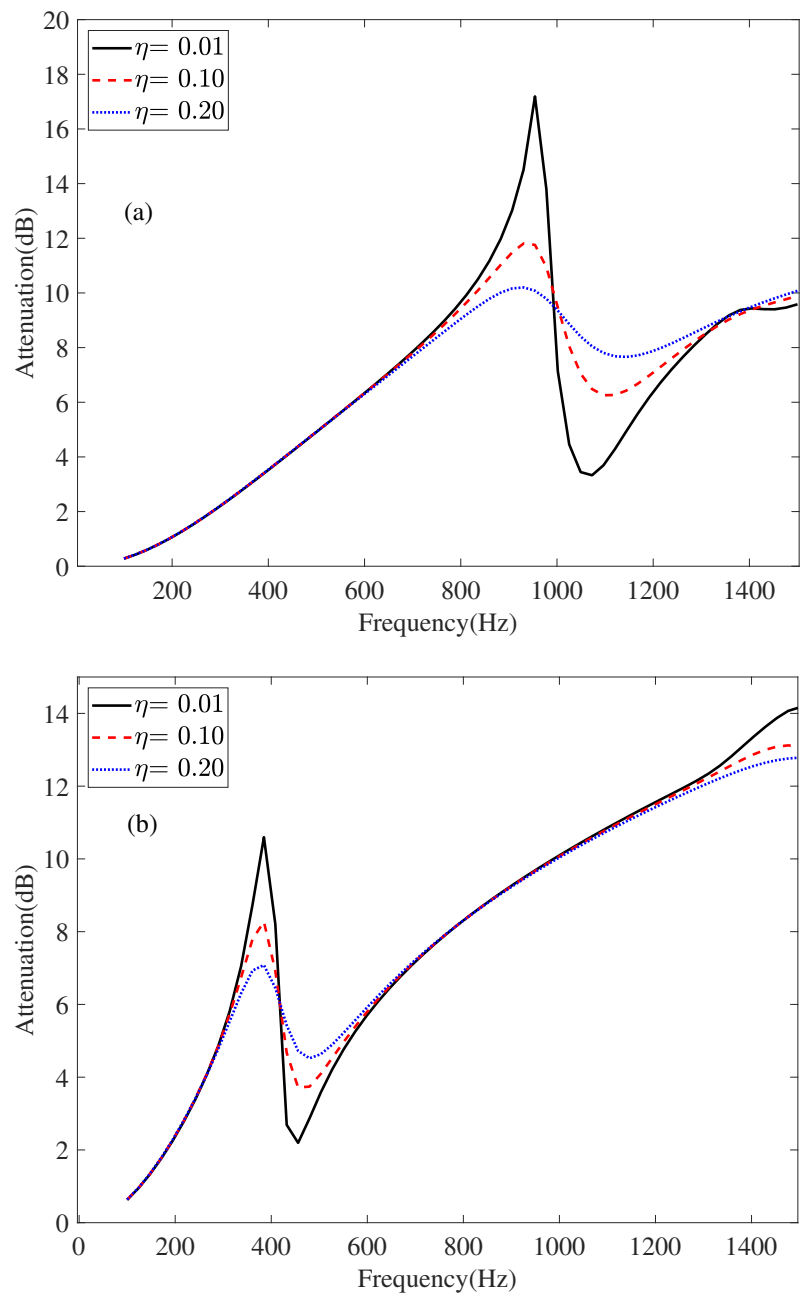

Figure 12: Attenuation of lamella network under different loss factors (LF) $[0.01,0.10,0.20]$. (a): parallel; (b): perpendicular.

ber, are nearly coalescing. The interested reader is referred to the seminal paper of Tester [23] for locally reacting materials and $[25,26]$ for rigid frame porous material and metamaterial having periodic structures. In the context of structural dynamics, this phenomenon is also known as veering and linked to the existence of exceptional points [38] which leads to strong attenuation $[39,40]$ due to the absence of beating phenomenon between both modes.

This is well illustrated for the perpendicular configuration in Fig. 15 where the evolution of the first four eigenvalues with respect to frequency are shown. It can be observed that maximal attenuation arises when the two least attenuated modes have wavenumbers which are getting closer in the complex plane. This happens around $400 \mathrm{~Hz}$ and also, to a least extent, around $1500 \mathrm{~Hz}$. One can also observe that 2 modes are nearly coalescing around $1100 \mathrm{~Hz}$ but with no effect on the TL since it does not affect the least attenuated mode. These situations correspond to strong modal interactions which are very sensitive to various parameters, geometrical and physical, especially those driving the coupling between the elastic frame and the fluid (see sec. 4.2) or influencing the frame resonances frequency (see 

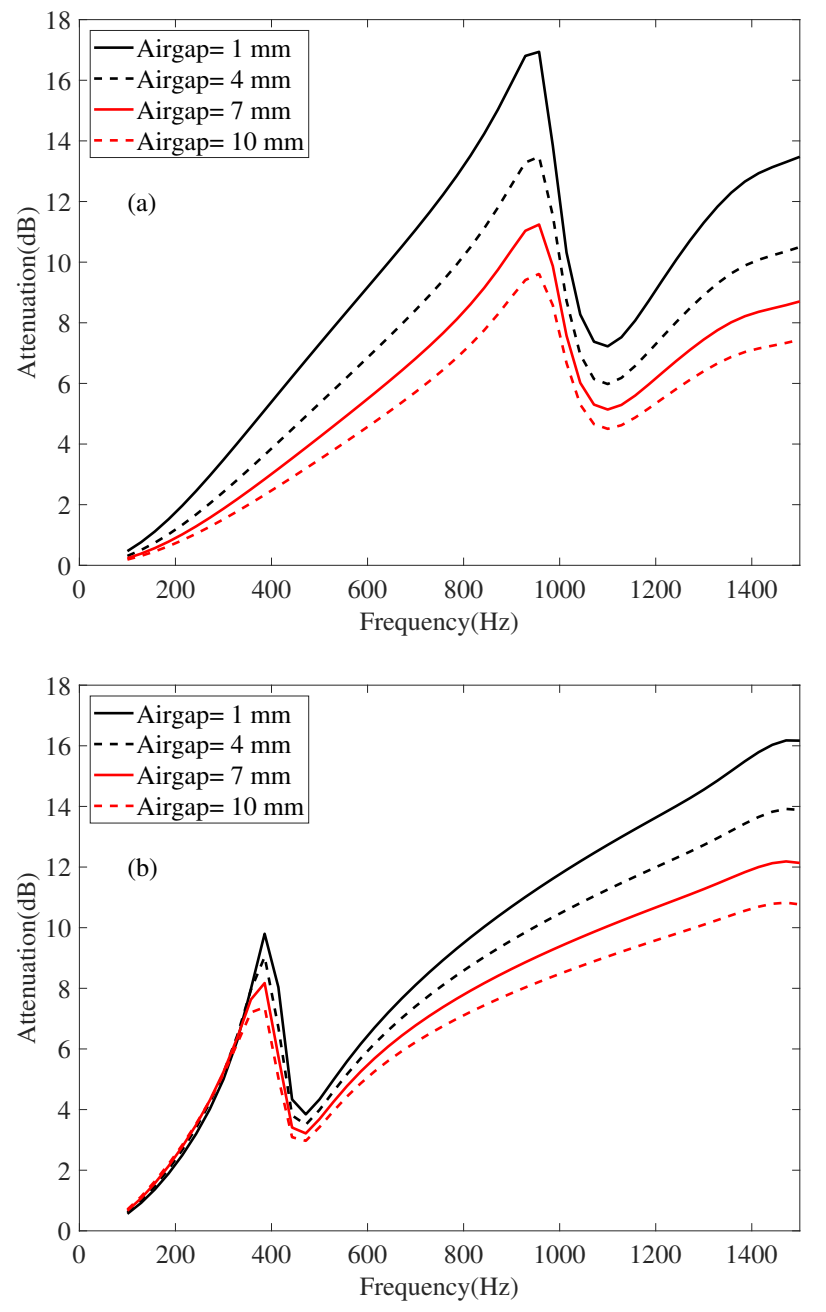

Figure 13: Attenuation of lamella network for different airgap width [1 mm, $4 \mathrm{~mm}, 7 \mathrm{~mm}, 10 \mathrm{~mm}$ ]. (a): parallel; (b): perpendicular.

sec.4.1). Finding optimized configurations could be achieved via parametric studies, as illustrated in this work, or by using optimization methods based on Exceptional point (EP) location as proposed in [26, 41].

\section{Conclusion}

The sound attenuation of a silencer consisting of a lamella network made with melamine foam inserted in rectangular duct is investigated numerically and experimentally. Two arrangements, parallel and perpendicular to the duct axis, are considered in the study. Results indicate that the perpendicular configuration yields strong low frequency sound attenuation peaks due to the excitation of bending modes whereas, in the parallel configuration, attenuation peaks are found to result from shear waves resonances across the width of the lamella. Though acoustic attenuation is mainly due to viscous dissipation as expected, the relative contribution from structural dissipation plays a noticeable role near the resonance.
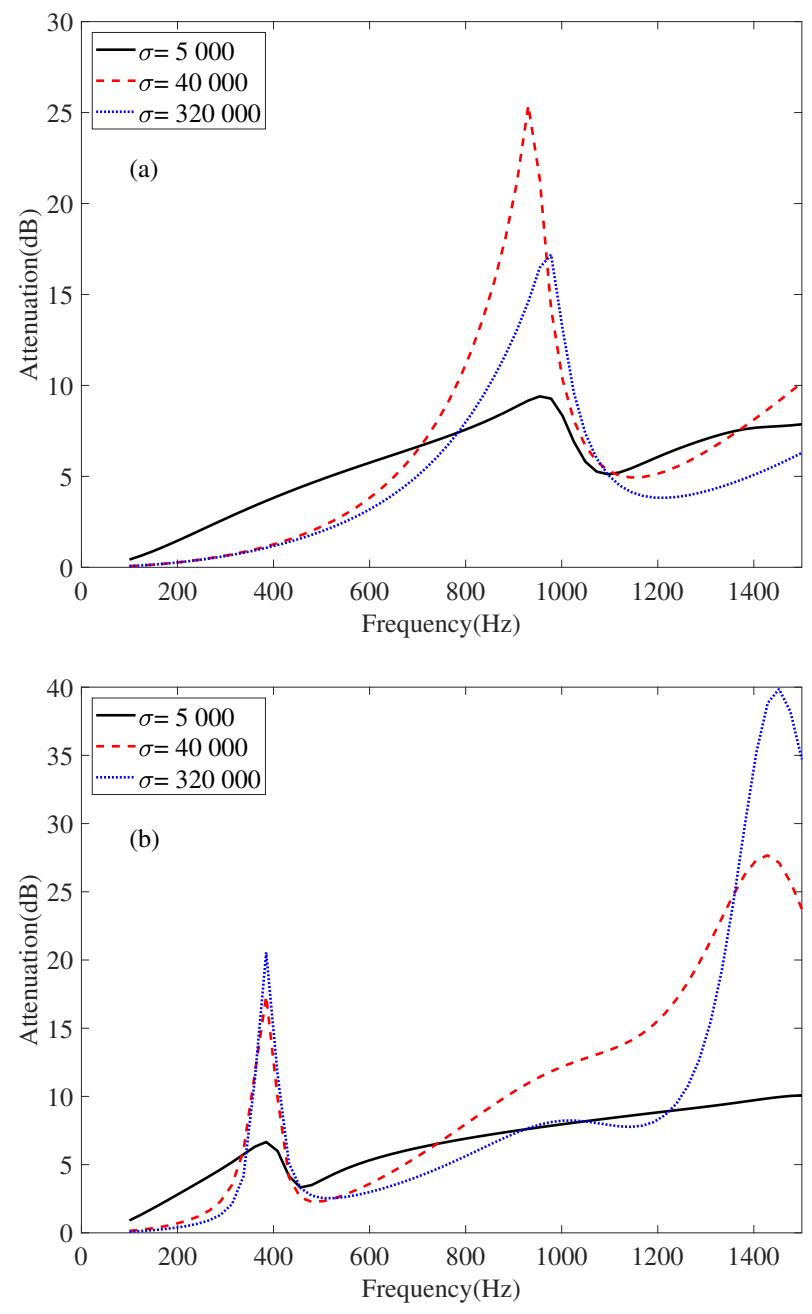

Figure 14: Comparison of TL performance of lamella network under different resistivity from 5000 to $320000 \mathrm{Nm}^{-4} \mathrm{~s}$. (a): parallel; (b): perpendicular.

The periodic structure of the silencer is exploited in order to devise a simplified numerical model which not only allows to diminish drastically the computational burden but also allows a more detailed analysis of the nature of the waves which propagate in the silencer. Because reflected waves at the entrance of the silencer can be neglected, this periodic model has been shown to be a reliable predictive tool showing good agreement with the full model and experimental results. A parametric study has been conducted in order to identify the effect of different geometrical parameters, i.e. dimensions of the lamella and air gap, as well as physical parameters, i.e. resistivity, elastic modulus of the porous foam and the loss factor, on the sound attenuation in the silencer. It is shown that resonance frequencies associated with peaks of attenuation, given by approximate formulas (Eq. (1) and (2) ), are proportional to the square root of the Young's modulus, and width of the lamella for the perpendicular configuration.

The interest for this new type of silencer concept relies mainly on the fact it can designed using relatively simple manufactur- 

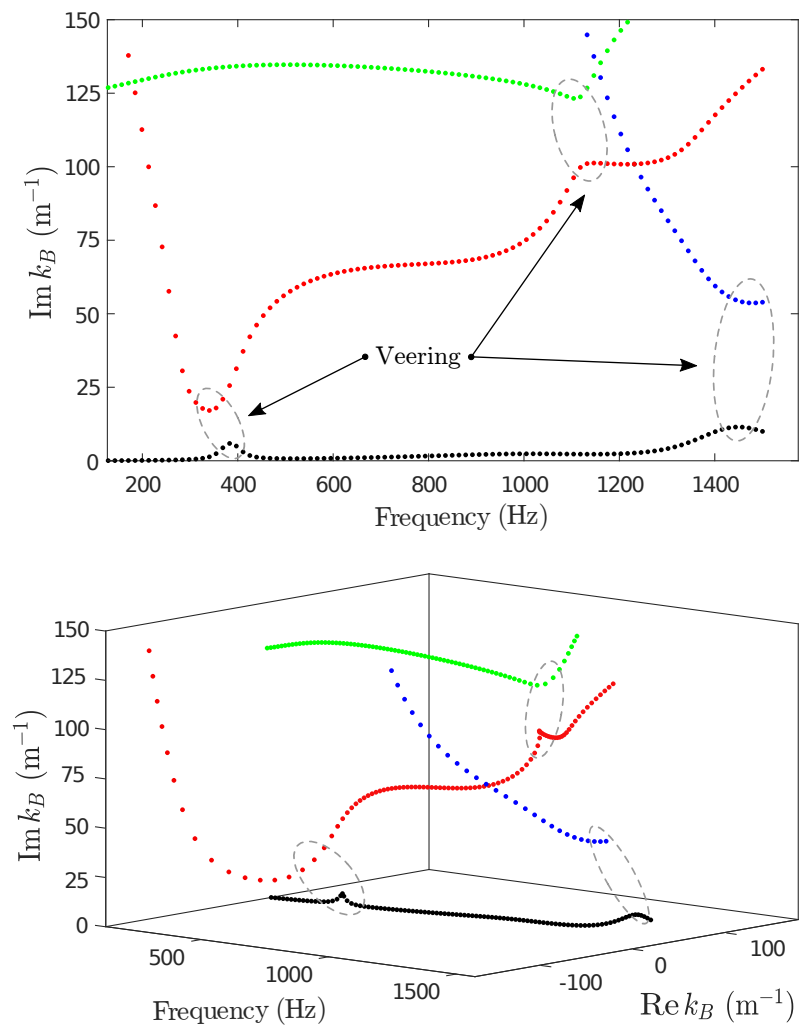

Figure 15: Bloch wavenumber on perpendicular case when $\sigma=$ $320000 \mathrm{Nm}^{-4} \mathrm{~s}$

ing processes without any other kind of inclusions. It is highly tunable and permits lower frequency attenuation, thanks to subwavelength resonances, whilst keeping its absorbing efficiency in the medium frequency range. It is thought that the concept could be investigated further by mixing several lamellas width or length in order to extend its efficiency in the low frequency regime.

\section{Acknowledgment}

This work was partially funded by the China Scholarship Council (No.201701810142).

\section{A. Rigid duct modes}

In the rigid duct, modal expansion is used in the DtN and to compute the TL. For instance, the transmitted pressure field reads

$$
p=\sum_{m=0}^{\infty} \sum_{n=0}^{\infty} A_{m n}^{t} \phi_{m n}(x, y) e^{\mathrm{i} k_{z, m n} z}
$$

where $A_{m n}^{t}$, represents the amplitude of the mode $(m, n)$. The upstream pressure is a combination of the incident and the reflected waves. For a rectangular duct, the orthonormal modal shape is given by

$$
\phi_{m n}=\Lambda_{m n} \cos \left(k_{x, m} x\right) \cos \left(k_{y, n} y\right)
$$

with $\Lambda_{m n}$ the modal norm [42], $k_{x, m}=m \pi / L_{x}, k_{y, n}=n \pi / L_{y}$ and $k_{z, m n}=\sqrt{k_{0}^{2}-k_{x, m}^{2}-k_{y, n}^{2}}$ are the wavenumbers in the $x, y$ and $z$ directions respectively. Once, the pressure field $p$ is known from the FEM computation, the amplitude of each transmitted mode can be recovered using orthogonality relation

$$
A_{m n}^{t}=\int_{\Gamma_{o}} p \phi_{m n} \mathrm{~d} \Gamma .
$$

The transmission loss can be obtained from

$$
T L=-10 \log _{10} \frac{I_{t}}{I_{i}}
$$

where $I_{i}$ and $I_{t}$ are the intensity of the incident and transmitted sound in duct respectively. The first is known and the later reads

$$
I_{t}=\sum_{m}^{M} \sum_{n=0}^{N} \frac{k_{z, m n}\left|A^{t}\right|_{m n}^{2}}{2 \omega \rho_{0}}
$$

where the sum is limited to the propagating modes.

\section{B. Weak formulation}

In the air domain, applying the standard weighted residual scheme to the Helmholtz Eq. (3) yields, after integrating by parts,

$$
-\int_{\Omega_{a}} \nabla p^{*} \cdot \nabla p \mathrm{~d} \Omega+k_{0}^{2} \int_{\Omega_{a}} p^{*} p \mathrm{~d} \Omega+\int_{\Gamma_{a}} p^{*} \frac{\partial p}{\partial n} \mathrm{~d} \Gamma=0,
$$

where $p^{*}$ stands for the weighting function. In the poroelastic domain governed by the Biot's Eqs. (4) expressed with $(\mathbf{u}, p)$, the same approach leads to [16, Chap. 13]

$$
\begin{gathered}
\int_{\Omega_{p}} \hat{\boldsymbol{\sigma}}^{s}(\mathbf{u}): \boldsymbol{\varepsilon}^{s}\left(\mathbf{u}^{*}\right) \mathrm{d} \Omega-\omega^{2} \int_{\Omega_{p}} \tilde{\rho} \mathbf{u} \cdot \mathbf{u}^{*} \mathrm{~d} \Omega \\
+\int_{\Omega_{p}}\left[\frac{\phi^{2}}{\omega^{2} \tilde{\rho}_{22}} \nabla p_{p} \cdot \nabla p_{p}^{*}-\frac{\phi^{2}}{\tilde{R}} p_{p} p_{p}^{*}\right] \mathrm{d} \Omega \\
-\int_{\Omega_{p}}\left(\tilde{\gamma}+\phi^{\prime}\right)\left(\nabla p_{p}^{*} \cdot \mathbf{u}+\nabla p_{p} \cdot \mathbf{u}^{*}\right) \mathrm{d} \Omega \\
\quad-\int_{\Omega_{p}} \phi^{\prime}\left(p_{p}^{*} \nabla \cdot \mathbf{u}+p_{p} \nabla \cdot \mathbf{u}^{*}\right) \mathrm{d} \Omega \\
-\int_{\Gamma_{p}} \boldsymbol{\sigma}^{t} \mathbf{n} \cdot \mathbf{u}^{*} \mathrm{~d} \Gamma-\int_{\Gamma_{p}} \phi(\mathbf{U}-\mathbf{u}) \cdot \mathbf{n} p_{p}^{*} \mathrm{~d} \Gamma=0 .
\end{gathered}
$$

with $\phi^{\prime}=\phi\left(1+\frac{Q}{R}\right)$.

\section{Weak formulation for Bloch waves computation}

We propose here a convenient systematic way to obtained the weak form of periodic part from the standard week formulation of each domain given in (B.1) and (B.2). The basic idea is to transform each differential operator to account for the Bloch decomposition given in Eq. (6). This approach can be managed automatically by picking all the combinations once the operator involving the pressure $p$ or $p_{p}$ and frame displacement $\mathbf{u}$ 
are expressed with the periodic fields $\hat{p}, \hat{p}_{p}$ and $\hat{\mathbf{u}}$. The advantage of this approach is to keep boundary terms unchanged which is convenient to applied standard boundary conditions like rigid wall. For instance gradient, divergence and vector gradient yield

$$
\begin{aligned}
\nabla p & =\left[\nabla \hat{p}+\mathrm{i} \hat{p} k_{B} \boldsymbol{\kappa}\right] e^{\mathrm{i} k_{B} \boldsymbol{\kappa} \cdot \mathbf{x}}, \\
\nabla \cdot \mathbf{u} & =\left[\nabla \cdot \hat{\mathbf{u}}+\mathrm{i} k_{B} \boldsymbol{\kappa} \cdot \hat{\mathbf{u}}\right] e^{\mathrm{i} k_{B} \boldsymbol{\kappa} \cdot \mathbf{x}}, \\
\nabla \hat{\mathbf{u}} & =\left[\boldsymbol{\nabla} \mathbf{u}+\mathrm{i} k_{B} \mathbf{u} \boldsymbol{\kappa}^{\mathrm{t}}\right] \mathrm{e}^{\mathrm{i} k_{B} \boldsymbol{\kappa} \cdot \mathbf{x}} .
\end{aligned}
$$

The strain tensor, using the Voight formalism

$$
\epsilon(\mathbf{u})=\left[\epsilon_{x x}, \epsilon_{y y}, \epsilon_{z z}, 2 \epsilon_{x y}, 2 \epsilon_{y z}, 2 \epsilon_{x z}\right]^{\mathrm{t}},
$$

now reads

$$
\epsilon(\mathbf{u})=\left[\mathbf{B}_{0}+k_{B} \mathbf{B}_{1}\right] \hat{\mathbf{u}} e^{\mathrm{i} k_{B} \kappa \cdot \mathbf{x}}
$$

where $\mathbf{B}_{0}$ and $\mathbf{B}_{1}$

$$
\mathbf{B}_{0}=\left[\begin{array}{ccc}
\partial_{x} & 0 & 0 \\
0 & \partial_{y} & 0 \\
0 & 0 & \partial_{z} \\
\partial_{y} & \partial_{x} & 0 \\
0 & \partial_{z} & \partial_{y} \\
\partial_{z} & 0 & \partial_{x}
\end{array}\right] \text { and } \quad \mathbf{B}_{1}=\mathrm{i}\left[\begin{array}{ccc}
\kappa_{x} & 0 & 0 \\
0 & \kappa_{y} & 0 \\
0 & 0 & \kappa_{z} \\
\kappa_{y} & \kappa_{x} & 0 \\
0 & \kappa_{z} & \kappa_{y} \\
\kappa_{z} & 0 & \kappa_{x}
\end{array}\right]
$$

In the air domain $\Omega_{a}$, this yields for volumic terms

$$
a_{0}\left(\hat{p}^{*}, \hat{p}\right)+k_{B} a_{1}\left(\hat{p}^{*}, \hat{p}\right)+k_{B}^{2} a_{2}\left(\hat{p}^{*}, \hat{p}\right)=0,
$$

with the operators

$$
\begin{aligned}
& a_{0}\left(\hat{p}^{*}, \hat{p}\right)=-\int_{\Omega_{a}} \nabla \hat{p}^{*} \cdot \nabla \hat{p} \mathrm{~d} \Omega+k_{a}^{2} \int_{\Omega_{a}} \hat{p}^{*} \hat{p} \mathrm{~d} \Omega, \\
& a_{1}\left(\hat{p}^{*}, \hat{p}\right)=\mathrm{i} \int_{\Omega_{a}}\left(-\nabla \hat{p}^{*} \cdot(\kappa \hat{p})+\left(\kappa \hat{p}^{*}\right) \cdot \nabla \hat{p}\right) \mathrm{d} \Omega, \\
& a_{2}\left(\hat{p}^{*}, \hat{p}\right)=-\int_{\Omega_{a}} \hat{p}^{*} \hat{p} \mathrm{~d} \Omega .
\end{aligned}
$$

In the poroelastic domain $\Omega_{p}$, this yields for volumic terms

$$
\begin{aligned}
b_{0}\left(\hat{p}_{p}^{*}, \hat{\mathbf{u}}^{*}, \hat{p}_{p}, \hat{\mathbf{u}}\right)+k_{B} b_{1}\left(\hat{p}_{p}^{*}, \hat{\mathbf{u}}^{*}, \hat{p}_{p}, \hat{\mathbf{u}}\right) \\
+k_{B}^{2} b_{2}\left(\hat{p}_{p}^{*}, \hat{\mathbf{u}}^{*}, \hat{p}_{p}, \hat{\mathbf{u}}\right)=0,
\end{aligned}
$$

with the operators

$$
\begin{aligned}
b_{0}\left(\hat{p}_{p}^{*}, \hat{\mathbf{u}}^{*}, \hat{p}_{p}, \hat{\mathbf{u}}\right) & =\int_{\Omega_{p}} \hat{\mathbf{u}}^{* t}\left(\mathbf{B}_{0}^{t} \mathbf{D} \mathbf{B}_{0}\right) \hat{\mathbf{u}} \mathrm{d} \Omega-\int_{\Omega_{p}} \tilde{\rho} \omega^{2} \hat{\mathbf{u}}^{*} \cdot \hat{\mathbf{u}} \mathrm{d} \Omega \\
& +\int_{\Omega_{p}}\left(\frac{\phi^{2}}{\omega^{2} \rho_{22}}\right) \nabla \hat{p}_{p}^{*} \cdot \nabla \hat{p}_{p} \mathrm{~d} \Omega-\int_{\Omega_{p}} \frac{\phi^{2}}{R} \hat{p}_{p}^{*} \hat{p}_{p} \mathrm{~d} \Omega \\
& -\int_{\Omega_{p}}\left(\gamma+\phi^{\prime}\right)\left(\nabla \hat{p}_{p}^{*} \cdot \hat{\mathbf{u}}+\hat{\mathbf{u}}^{*} \cdot \nabla \hat{p}_{p}\right) \mathrm{d} \Omega \\
& -\int_{\Omega_{p}} \phi^{\prime}\left(\hat{p}_{p}^{*} \nabla \cdot \hat{\mathbf{u}}+\nabla \cdot \hat{\mathbf{u}}^{*} \hat{p}_{p}\right) \mathrm{d} \Omega,
\end{aligned}
$$

$$
\begin{aligned}
b_{1}\left(\hat{p}_{p}^{*}, \hat{\mathbf{u}}^{*}, \hat{p}_{p}, \hat{\mathbf{u}}\right) & =\int_{\Omega_{p}} \hat{\mathbf{u}}^{* t}\left(\mathbf{B}_{0}^{t} \mathbf{D} \mathbf{B}_{1}-\mathbf{B}_{1}^{t} \mathbf{D} \mathbf{B}_{0}\right) \hat{\mathbf{u}} \mathrm{d} \Omega \\
& +\int_{\Omega_{p}} \frac{\phi^{2}}{\omega^{2} \rho_{22}}\left(\nabla \hat{p}_{p}^{*} \cdot \boldsymbol{\kappa} \mathrm{i} \hat{p}_{p}-\hat{p}_{p}^{*} \mathrm{i} \boldsymbol{\kappa} \cdot \nabla \hat{p}_{p}\right) \mathrm{d} \Omega \\
& -\left(\gamma+\phi^{\prime}\right) \int_{\Omega_{p}}-\hat{p}_{p}^{*} \mathrm{i} \boldsymbol{\kappa} \cdot \hat{\mathbf{u}}+\hat{\mathbf{u}}^{*} \cdot \boldsymbol{\kappa} \mathrm{i} \hat{p}_{p} \mathrm{~d} \Omega \\
& -\phi^{\prime} \int_{\Omega_{p}} \hat{p}_{p}^{*} \mathrm{i} \boldsymbol{\kappa} \cdot \hat{\mathbf{u}}-\hat{\mathbf{u}}^{*} \cdot \boldsymbol{\kappa} \mathrm{i} \hat{p}_{p} \mathrm{~d} \Omega
\end{aligned}
$$

$$
\begin{aligned}
b_{2}\left(\hat{p}_{p}^{*}, \hat{\mathbf{u}}^{*}, \hat{p}_{p}, \hat{\mathbf{u}}\right) & =-\int_{\Omega_{p}} \hat{\mathbf{u}}^{* t} \mathbf{B}_{1}^{t} \mathbf{D} \mathbf{B}_{1} \hat{\mathbf{u}} \mathrm{d} \Omega \\
& +\int_{\Omega_{p}}\left(\frac{\phi^{2}}{\omega^{2} \rho_{22}}\right) \hat{p}_{p}^{*}(\boldsymbol{\kappa} \cdot \boldsymbol{\kappa}) \hat{p}_{p} \mathrm{~d} \Omega .
\end{aligned}
$$

Once the boundary conditions are applied, the discrete operator (C.8) and (C.9) are assembled and coupled with the condition given in sec. 3 , the quadratic eigenvalue problem of whole model (8) can be found.

\section{References}

[1] R. Kirby, P. T. Williams, J. Hill, A three dimensional investigation into the acoustic performance of dissipative splitter silencers, J. Acoust. Soc. Am. 135 (5) (2014) 2727-2737. doi:10.1121/1.4869089.

[2] R. Binois, E. Perrey-Debain, N. Dauchez, B. Nennig, J.-M. Ville, G. Beillard, On the efficiency of parallel baffle-type silencers in rectangular ducts: prediction and measurement, Acta Acust. United Ac. 101 (2015) 520-530. doi: 10.3813/AAA .918849.

[3] B. Nennig, R. Binois, E. Perrey-Debain, N. Dauchez, A homogenization method used to predict the performance of silencers containing parallel splitters, J. Acoust. Soc. Am. 137 (6) (2015) 3221-31. doi:10.1121/ 1. 4921598.

[4] Z. Yang, J. Mei, M. Yang, N. H. Chan, P. Sheng, Membrane-type acoustic metamaterial with negative dynamic mass, Phys. Rev. Lett. 101 (20). doi:10.1103/PhysRevLett.101.204301.

[5] N. Fang, D. Xi, J. Xu, M. Ambati, W. Srituravanich, C. Sun, X. Zhang, Ultrasonic metamaterials with negative modulus, Nat. Mater. 5 (6) (2006) 452-456. doi:10.1038/nmat1644.

[6] X. Hu, K.-M. Ho, C. T. Chan, J. Zi, Homogenization of acoustic metamaterials of Helmholtz resonators in fluid, Phys.1 Rev. B 62 (2008) 30. doi:10.1103/PhysRevB.77.172301.

[7] Y. Li, B. M. Assouar, Acoustic metasurface-based perfect absorber with deep subwavelength thickness, Appl. Phys. Lett. 108 (6). doi:10.1063/ 1. 4941338.

[8] Z. Liang, J. Li, Extreme acoustic metamaterial by coiling up space, Phys. Rev. Lett. 108 (11). doi:10.1103/PhysRevLett.108.114301.

[9] X. Ni, Y. Wu, Z. G. Chen, L. Y. Zheng, Y. L. Xu, P. Nayar, X. P. Liu, M. H. Lu, Y. F. Chen, Acoustic rainbow trapping by coiling up space, Sci. Rep. 4 (2014) 7038. doi:10.1038/srep07038.

[10] X. Cai, Q. Guo, G. Hu, J. Yang, Ultrathin low-frequency sound absorbing panels based on coplanar spiral tubes or coplanar Helmholtz resonators, Appl. Phys. Lett. 105 (12). doi: 10.1063/1. 4895617.

[11] T. Yamamoto, Acoustic metamaterial plate embedded with Helmholtz resonators for extraordinary sound transmission loss, J. Appl. Phys. 123 (21). doi : $10.1063 / 1.5025570$.

[12] J.-P. Groby, C. Lagarrigue, B. Brouard, O. Dazel, V. Tournat, B. Nennig, Enhancing the absorption properties of acoustic porous plates by periodically embedding Helmholtz resonators, J. Acoust. Soc. Am. 137 (1) (2015) 273-280. doi : 10.1121/1.4904534.

[13] V. Romero-García, G. Theocharis, O. Richoux, A. Merkel, V. Tournat, V. Pagneux, Perfect and broadband acoustic absorption by critically coupled sub-wavelength resonators, Sci. Rep. 6 (2016) 19519. doi: $10.1038 /$ srep 19519. 
[14] P. Leclaire, O. Umnova, T. Dupont, R. Panneton, Acoustical properties of air-saturated porous material with periodically distributed dead-end pores, J. Acoust. Soc. Am. 137 (4) (2015) 1772-1782. doi:10.1121/ 1.4916712 .

[15] N. Jiménez, V. Romero-García, V. Pagneux, J.-P. Groby, Quasiperfect absorption by subwavelength acoustic panels in transmission using accumulation of resonances due to slow sound, Phys. Rev. B 95 (1) (2017) 014205. doi:10.1103/PhysRevB.95.014205.

[16] J. F. Allard, N. Atalla, Propagation of Sound in Porous Media: Modelling Sound Absorbing Materials, John Wiley and Sons, 2009. doi :10.1002/ 9780470747339

[17] C. Boutin, Acoustics of porous media with inner resonators, J. Acoust. Soc. Am. 134 (6) (2013) 4717-4729. doi : 10.1121/1.4824965.

[18] J. Yang, J. S. Lee, Y. Y. Kim, Metaporous layer to overcome the thickness constraint for broadband sound absorption, J. Appl. Phys. 117 (17) (2015) 174903. doi:10.1063/1.4919844.

[19] J. Yang, J. S. Lee, Y. Y. Kim, Multiple slow waves in metaporous layers for broadband sound absorption, J. Phys. D 50 (1) (2016) 015301. doi : 10. 1088/1361-6463/50/1/015301.

[20] S. Griffiths, B. Nennig, S. Job, Porogranular materials composed of elastic helmholtz resonators for acoustic wave absorption, J. Acoust. Soc. Am 141 (1) (2017) 254-264. doi : 10.1121/1.4973691.

[21] J. Christensen, V. Romero-García, R. Picó, A. Cebrecos, F. J. De Abajo, N. A. Mortensen, M. Willatzen, V. J. Sánchez-Morcillo, Extraordinary absorption of sound in porous lamella-crystals, Sci. Rep. 4. doi:10. $1038 /$ srep04674.

[22] N. Dauchez, B. Nennig, O. Robin, Additional Sound Absorption Within a Poroelastic Lamella Network Under Oblique Incidence, Acta Acust. United Ac 104 (2) (2018) 211-219. doi : 10.3813/AAA.919162 Ã́r.

[23] B. J. Tester, The optimization of modal sound attenuation in duct, in the absence of mean flow, J. Sound Vib. 27 (1973) 477-513. doi:10.1016/ S0022-460X (73) $80358-X$.

[24] Y. Aurégan, M. Farooqui, J.-P. Groby, Low frequency sound attenuation in a flow duct using a thin slow sound material, The Journal of the Acoustical Society of America 139 (5) (2016) EL149-EL153. arXiv : https : //doi.org/10.1121/1.4951028, doi:10.1121/1.4951028. URL https : //doi.org/10.1121/1.4951028

[25] L. Xiong, B. Nennig, Y. Aurégan, W. Bi, Sound attenuation optimization using metaporous materials tuned on exceptional points, J. Acoust. Soc. Am. 142 (4) (2017) 2288-2297. doi:10.1121/1.5007851.

[26] X. Qiu, X. Jing, L. Du, X. Sun, M. Åbom, H. Bodén, Mode-merging design method for nonlocally reacting liners with porous materials, AIAA Journal (2020) 1-13doi: 10.2514/1. J058958.

[27] L. Xiong, W. Bi, Y. Aurégan, Fano resonance scatterings in waveguides with impedance boundary conditions, J. Acoust. Soc. Am. 139 (2) (2016) 764-772. doi: 10.1121/1.4941568.

[28] R. Ghaffarivardavagh, J. Nikolajczyk, S. Anderson, X. Zhang, Ultra-open acoustic metamaterial silencer based on fano-like interference, Phys. Rev. B 99 (2) (2019) 024302. doi:10.1103/physrevb.99.024302.

[29] B. Nennig, E. Perrey-Debain, M. Ben Tahar, A mode matching method for modelling dissipative silencers lined with poroelastic materials and containing mean flow, J. Acoust. Soc. Am. 128 (6) (2010) 3308-3320. doi:10.1121/1.3506346.

[30] B. Nennig, M. Ben Tahar, E. Perrey-Debain, A displacement-pressure finite element formulation for analyzing the sound transmission in ducted shear flows with finite poroelastic lining, J. Acoust. Soc. Am. 130 (1) (2011) 42-51. doi: 10.1121/1.3598451.

[31] C. Jiang, C. Wang, L. Huang, Acoustic characterization of ducts lined with poroelastic materials based on wave finite element method, Appl. Acoust. 145 (2019) 362-373. doi:10.1016/j.apacoust.2018.10. 030 .

[32] H. Trabelsi, N. Zerbib, J. M. Ville, F. Foucart, Passive and active acoustic properties of a diaphragm at low Mach number, Experimental procedure and numerical simulation, Eur. J. Comp. Mech. 20 (1-4) (2011) 49-71. doi:10.3166/EJCM.20.49-71.

[33] L. Boeckx, P. Leclaire, P. Khurana, C. Glorieux, W. Lauriks, J.-F. Allard, Investigation of the phase velocities of guided acoustic waves in soft porous layers, J. Acoust. Soc. Am. 117 (2) (2005) 545-554. doi : 10.1121/1.1847848.

[34] I. Harari, I. Patlashenko, D. Givoli, Dirichlet-to-neumann maps for unbounded wave guides, J. Comp. Phys. 143 (1) (1998) $200-223$. doi : 10.1006/jcph.1998.5960.

[35] M. Collet, M. Ouisse, M. Ruzzene, M. Ichchou, Floquet-Bloch decomposition for the computation of dispersion of two-dimensional periodic, damped mechanical systems, Int. J. Solids Struct. 48 (20) (2011) 2837 2848. doi: 10.1016/j.ijsolstr.2011.06.002.

[36] O. Doutres, N. Dauchez, J.-M. Genevaux, Porous layer impedance applied to a moving wall: Application to the radiation of a covered piston, J. Acoust. Soc. Am. 121 (1) (2007) 206-213. doi:10.1121/1.2359233.

[37] O. Dazel, F. Sgard, F.-X. Becot, N. Atalla, Expressions of dissipated powers and stored energies in poroelastic media modeled by $\{u, U\}$, and $\{u, P\}$ formulations, J. Acoust. Soc. Am. (2008) 2054-2063doi : 10.1121/1. 2874520.

[38] M. Ghienne, B. Nennig, Beyond the limitations of perturbation methods for real random eigenvalue problems using exceptional points and analytic continuation, J. Sound Vib. (2020) 115398doi:10.1016/j.jsv. 2020.115398.

[39] J.-W. Ryu, W.-S. Son, D.-U. Hwang, S.-Y. Lee, S. W. Kim, Exceptional points in coupled dissipative dynamical systems, Phy. Rev. E 91 (5) (2015) 052910. doi:10.1103/physreve.91.052910.

[40] G. Dolfo, J. Vigué, Damping of coupled harmonic oscillators, Eur. J. Phys. 39 (2) (2018) 025005. doi : 10.1088/1361-6404/aa9ec6.

[41] B. Nennig, E. Perrey-Debain, A high order continuation method to locate exceptional points and to compute puiseux series with applications to acoustic waveguides, J. Comp. Phys. (2020) 109425doi : 10.1016/j . jcp. 2020.109425.

[42] P. M. Morse, K. U. Ingard, Theoretical acoustics, Princeton university press, 1986. 